\title{
Registration and Analysis of White Matter Group Differences with a Multi-fiber Model
}

\author{
Maxime Taquet ${ }^{1,2}$, Benoît Scherrer ${ }^{1}$, Olivier Commowick ${ }^{3}$, Jurriaan Peters ${ }^{1,4}$, \\ Mustafa Sahin ${ }^{4}$, Benoît Macq ${ }^{2}$, and Simon K. Warfield ${ }^{1}$ \\ ${ }^{1}$ Computational Radiology Laboratory, Children's Hospital Boston, Harvard, USA \\ 2 ICTEAM Institute, Université Catholique de Louvain, Louvain-La-Neuve, Belgium \\ 3 INRIA, INSERM, VisAGeS U746 Unit/Project, F-35042 Rennes, France \\ ${ }^{4}$ Department of Neurology, Children's Hospital Boston, Harvard, USA
}

\begin{abstract}
Diffusion magnetic resonance imaging has been used extensively to probe the white matter in vivo. Typically, the raw diffusion images are used to reconstruct a diffusion tensor image (DTI). The incapacity of DTI to represent crossing fibers leaded to the development of more sophisticated diffusion models. Among them, multi-fiber models represent each fiber bundle independently, allowing the direct extraction of diffusion features for population analysis. However, no method exists to properly register multi-fiber models, seriously limiting their use in group comparisons. This paper presents a registration and atlas construction method for multi-fiber models. The validity of the registration is demonstrated on a dataset of 45 subjects, including both healthy and unhealthy subjects. Morphometry analysis and tract-based statistics are then carried out, proving that multi-fiber models registration is better at detecting white matter local differences than single tensor registration.
\end{abstract}

Keywords: Diffusion Imaging, Multi-Fiber Models, Registration, White Matter.

\section{Introduction}

Diffusion magnetic resonance imaging offers the ability to investigate in vivo the white matter microstructure. The representation of the signal by diffusion tensor images (DTI) has proven useful for population analysis in two ways [1]. First, scalar features extracted from DTI, such as the fractional anisotropy (FA), may indicate the presence of brain diseases. Second, the use of DTI in registration improves the detection of morphometric differences, compared to scalar images.

The single tensor diffusion model has, however, proven inaccurate for two main reasons. First, it cannot represent the signal arising from multiple fibers with heterogeneous orientations in one voxel. Second, it does not account for the non-monoexponential decay observed when imaging at high b-values. Novel models addressing one or both of these issues have been introduced [2] : Qball imaging, spherical deconvolution, 4th order tensors, DOT, and others. Most of them focus on describing the general shape of the diffusion at each voxel. 
In contrast, mixture models represent each fiber bundle independently, keeping the interpretability of single fiber models while accounting for crossing fibers. Therefore, scalar quantities such as the fractional anisotropy (FA) can still be computed for each fiber independently. This property makes them very attractive for population analysis.

While the literature on the registration of complex diffusion models is growing (e.g., 34]), no method has been developed to register mixture models. This lack of a registration method limits the use of mixture models in population analysis despite their attractiveness. This issue has been previously reported, and has incited researches to register the raw diffusion weighted images instead [5].

The remaining of this paper is organized as follows. Section 2 introduces the diffusion mixture models. Section 3 presents a method to compute weighted average of mixture models. Section 4 develops a similarity metric for diffusion mixture images. Section 5 presents the integration of the developed methods in a registration algorithm and analyzes its complexity. Section 6 presents experimental results on a dataset of 45 subjects. Finally, Section 7 concludes.

\section{Diffusion Mixtures}

The basic idea behind multi-fiber models is to fit a single fiber model to each of the fiber bundles present in the voxel. If $S_{i}(\boldsymbol{x})$ is a suitable model to represent the diffusion process in a single fiber, then,

$$
S(\boldsymbol{x})=\sum_{i=1}^{N} f_{i} S_{i}(\boldsymbol{x})
$$

is a multi-fiber model for $N$ crossing fibers with relative volumetric occupancy given by $f_{i}$. The assumption behind these models is that the exchange of water molecules between populations of fibers is negligible during the diffusion time [2].

The simplest multi-fiber model is the multi-tensor model in which $S_{i}=$ $S_{0} e^{-b \boldsymbol{g}^{T} \boldsymbol{D}_{i} \boldsymbol{g}}$. More complex multi-fiber models have later been introduced 6 . Potentially, any single fiber model can be extended to a multi-fiber model by means of mixtures. One such model, the biexponential decay model [2], represents each fiber bundle by a Gaussian mixture to capture the non-monoexponential decay of the signal. The corresponding multi-fiber model would be a mixture of Gaussian mixtures which is itself a Gaussian mixture. A natural parameterization of diffusion Gaussian mixtures is the set of pairs (fraction, covariance matrix), that we write: $\left\{\left(f_{1}, \boldsymbol{\Sigma}_{1}\right), \ldots,\left(f_{N}, \boldsymbol{\Sigma}_{N}\right)\right\}$. Alternatively, to connect with the tensor formalism, the inverse of the covariance matrix, $\boldsymbol{D}_{i}=\boldsymbol{\Sigma}_{i}^{-1}$, can be used.

\section{Weighted Combination of Mixtures}

Computing weighted combinations of voxel values is at the basics of interpolation (the value in one location is the weighted combination of the values in the 
neighborhood), smoothing (the value at a grid voxel is replaced by a weighted combination of the values in a neighborhood) and atlas construction (the value at one voxel is the average of the values in the aligned subjects').

Gaussian mixture simplification (GMS) was introduced to efficiently compute weighted combinations of diffusion mixture [7]. In this section, we underline the important aspects of this method. The idea behind GMS is that computing weighted combinations of mixtures would be trivial if the number of components of the result could be arbitrarily large. Indeed, the linear combination of $K$ mixture models with $N$ components is a mixture models with $M=K N$ components:

$$
\mathcal{M}_{C}=\sum_{k=1}^{K} w_{k} \mathcal{M}_{k}=\sum_{k=1}^{K} w_{k} \sum_{j=1}^{N} f_{j}^{k} S_{j}^{k}(\boldsymbol{x}) \equiv \sum_{i=1}^{M} g_{i} S_{i}(\boldsymbol{x}) .
$$

We refer to this mixture as the complete mixture. GMS optimizes the parameters of a simplified mixture $\mathcal{M}_{S}=\sum_{j=1}^{N} h_{j} R_{j}(\boldsymbol{x})$ with $N<M$ components that best approximates $\mathcal{M}_{C}$. The energy function to be minimized is the cumulative differential entropy (the reference to the location $\boldsymbol{x}$ is omitted for clarity):

$$
D\left(\mathcal{M}_{C}, \mathcal{M}_{S}\right)=\sum_{j=1}^{N} \sum_{i: \pi_{i}=j} g_{i} D\left(S_{i} \| R_{j}\right)=\sum_{j=1}^{N} \sum_{i: \pi_{i}=j} g_{i} \int S_{i}(\boldsymbol{g}) \log \frac{S_{i}(\boldsymbol{g})}{R_{j}(\boldsymbol{g})} d \boldsymbol{g}
$$

where $\boldsymbol{g}$ is the gradient vector and where latent variables $\pi_{i}$ cluster the components of the complete mixture $S_{i}$ in $N$ clusters each represented by a single component of the simplified mixture, $R_{j} ; \pi_{i}=j$ means that $S_{i}$ is best represented by $R_{j}$. Following the recent developments in probabilistic clustering, an EM scheme is used to minimize (3). Banerjee et al showed that both the E-step and the M-step can be solved in closed form for mixtures of exponential distributions 8. For Gaussian mixtures, the E-step consists in optimizing the latent variables $\pi_{i}$ by computing the Burg matrix divergence between the covariance matrices of each component of $\mathcal{M}_{C}\left(\boldsymbol{\Sigma}_{i}^{S}\right)$ and each component of $\mathcal{M}_{S}\left(\boldsymbol{\Sigma}_{j}^{R}\right)$ :

$$
\pi_{i}=\underset{j}{\arg \min } B\left(\boldsymbol{\Sigma}_{i}^{S}, \boldsymbol{\Sigma}_{j}^{R}\right)=\underset{j}{\arg \min } \operatorname{Tr}\left(\boldsymbol{\Sigma}_{i}^{S} \boldsymbol{\Sigma}_{j}^{R^{-1}}\right)-\log \left|\boldsymbol{\Sigma}_{i}^{S} \boldsymbol{\Sigma}_{j}^{R^{-1}}\right| .
$$

As for the M-step, it sums up to calculating:

$$
\boldsymbol{\Sigma}_{j}^{R}=\frac{\sum_{i: \pi_{i}=j} f_{i} \boldsymbol{\Sigma}_{i}^{S}}{\sum_{i: \pi_{i}=j} f_{i}} \quad \text { and } \quad h_{j}=\sum_{i: \pi_{i}=j} f_{i} .
$$

Alternating (4) and (5) until convergence provides the parameters $\left(h_{j}\right.$ and $\left.\boldsymbol{\Sigma}_{j}^{R}\right)$ of the resulting mixture. A log-Euclidean version of this interpolation scheme is obtained by replacing all covariance matrices by their logarithm.

\section{Generalized Correlation Coefficient for Mixtures}

The correlation coefficient, invariant under linear transformations of the voxel intensities, is widely used in mono-modal image registration. The inter-subject 

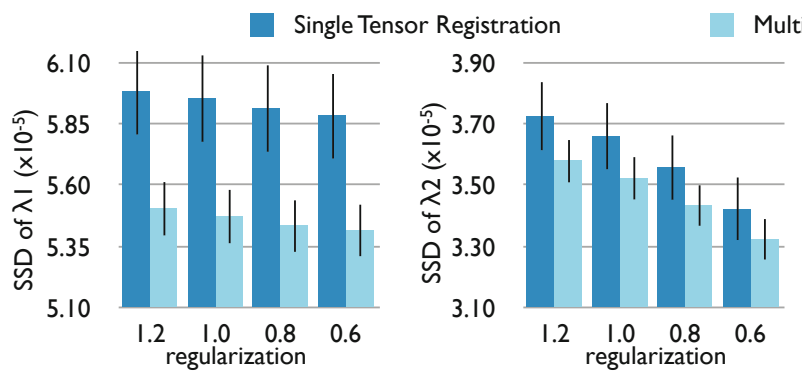

Multi-Fiber Registration

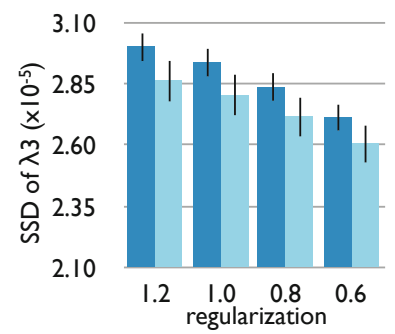

Fig. 1. Comparison of the single tensor and multi-fiber registration in terms of the SSD between eigenvalues after alignment, for different regularization parameter values [9]. Multi-fiber registration significantly improves the quality of the registration.

variability of diffusivity values motivates the introduction of a generalized correlation coefficient, invariant under these differences. In DTI, this variability has been reported and partially accounted for in some registration methods [1]. The correlation coefficient between blocks $F$ and $G$ is defined as the scalar product of the normalized blocks:

$$
\rho(F, G)=\left\langle\frac{F-\mu_{F}}{\left\|F-\mu_{F}\right\|}, \frac{G-\mu_{G}}{\left\|G-\mu_{G}\right\|}\right\rangle,
$$

where $\mu_{F}$ is the mean of the image values in the block. It is invariant if $F$ (and/or $G$ ) is replaced by $a F+b$. It has been generalized to vector images by redefining the means $\mu_{F}$ and $\mu_{G}$ as the projection of the block onto a constant block $T$ [10]:

$$
F-\mu_{F}=F-\langle F, T\rangle \frac{T}{\|T\|^{2}} .
$$

The corresponding generalized correlation coefficient is invariant if $F$ is replaced by $a F+b T$ where $T$ is now any constant vector block. The definition of a scalar product between two blocks of mixture models seems impractical if not impossible. We therefore further generalize the correlation coefficient by substituting the inner product by a more general scalar mapping, $m\left(\mathcal{M}_{f}, \mathcal{M}_{g}\right)$ :

$$
\rho\left(\mathcal{M}_{f}, \mathcal{M}_{g}\right)=m\left(\frac{\mathcal{M}_{f}-m\left(\mathcal{M}_{f}, \mathcal{T}\right) \mathcal{T}}{n_{m}\left(\mathcal{M}_{f}-m\left(\mathcal{M}_{f}, \mathcal{T}\right) \mathcal{T}\right)}, \frac{\mathcal{M}_{g}-m\left(\mathcal{M}_{g}, \mathcal{T}\right) \mathcal{T}}{n_{m}\left(\mathcal{M}_{g}-m\left(\mathcal{M}_{g}, \mathcal{T}\right) \mathcal{T}\right)}\right)
$$

where $n_{m}(\mathcal{M})^{2}=m(\mathcal{M}, \mathcal{M})$ is a generalization of the norm. This definition does not guarantee the invariance property of the metric for any scalar mapping. One can show that the invariance is preserved as long as the scalar mapping is linear with respect to the constant block $\mathcal{T}$ :

$$
m\left(a \mathcal{M}_{f}+b \mathcal{T}, \mathcal{T}\right)=a m\left(\mathcal{M}_{f}, \mathcal{T}\right)+b m(\mathcal{T}, \mathcal{T})
$$

To preserve the interpretability of $\rho$ as a similarity metric, it needs to be symmetric, equal to one in case of perfect match and lower than one in any other case. These constraints on $\rho$ translate into the following constraints on $m$ : 


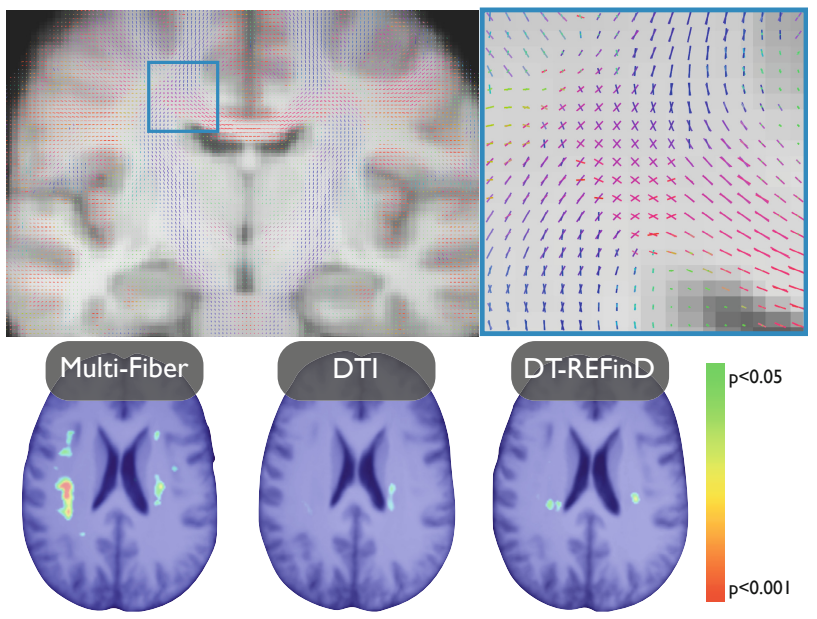

Fig. 2. (top) The two-tensor atlas built by means of the developed registration method reveals crossing pathways common to all anatomies. (bottom) White matter volume shrinkage in tuberous sclerosis represented by the p-value maps. Multi-fiber registration reveals more differences than single tensor registration and DT-REFinD 11 .

$$
\begin{aligned}
m\left(\mathcal{M}_{f}, \mathcal{M}_{g}\right) & =m\left(\mathcal{M}_{g}, \mathcal{M}_{f}\right) \\
n_{m}\left(a \mathcal{M}_{f}\right) & =a n_{m}\left(\mathcal{M}_{f}\right) \\
\left|m\left(\mathcal{M}_{f}, \mathcal{M}_{g}\right)\right| & \leq n_{m}\left(\mathcal{M}_{f}\right) n_{m}\left(\mathcal{M}_{g}\right) .
\end{aligned}
$$

The latter is a generalized form of the Cauchy-Schwartz inequality for inner products. Conditions (6 9), the choice of $\mathcal{T}$ and the definitions of the addition $\mathcal{M}+\mathcal{T}$ and multiplication by a scalar $a \mathcal{M}$, stand together as a model to define a correlation coefficients in potentially any space. For DTI, if $\mathcal{T}$ is an isotropic tensor block $\left(\mathcal{T}(\boldsymbol{x})=D \boldsymbol{I}_{3 \times 3}\right), m$ is the log-Euclidean scalar product, and the log-Euclidean algebra is used, then $\rho$ is invariant under linear transformations of the eigenvalues in the log-domain [12. For multi-tensor images, we fix $\mathcal{T}(\boldsymbol{x})=\left\{\left(\frac{1}{N}, D \boldsymbol{I}_{3 \times 3}\right), \ldots,\left(\frac{1}{N}, D \boldsymbol{I}_{3 \times 3}\right)\right\}$, and we define the addition of $\mathcal{T}$, and the multiplication by a scalar component-wise in the log-domain. The scalar mapping $m\left(\mathcal{M}_{f}, \mathcal{M}_{g}\right)$ is defined by pairing the tensors in each voxel to maximize the linear combination of pairwise scalar products. Let $\mathcal{M}_{f}(\boldsymbol{x})=\left\{\left(f_{1}, \boldsymbol{F}_{1}\right), \ldots,\left(f_{N}, \boldsymbol{F}_{N}\right)\right\}$ and $\mathcal{M}_{g}(\boldsymbol{x})=\left\{\left(g_{1}, \boldsymbol{G}_{1}\right), \ldots,\left(g_{N}, \boldsymbol{G}_{N}\right)\right\}$ defined on a domain $\Omega$, we have:

$$
m\left(\mathcal{M}_{f}, \mathcal{M}_{g}\right)=\sum_{\boldsymbol{x} \in \Omega} \max _{\pi} \sum_{i=1}^{N} f_{i} g_{\pi(i)}\left\langle\boldsymbol{F}_{i}, \boldsymbol{G}_{\pi(i)}\right\rangle,
$$

where $\pi$ is a pairing function associating one tensor of $\mathcal{M}_{g}$ to each tensor of $\mathcal{M}_{f}$. This scalar mapping satisfies conditions (6-9). Interestingly, the resulting generalized correlation coefficient is invariant under any global (within the block) linear transformation of all eigenvalues in the log-domain. This similarity metric is therefore robust to the inter-subject variability of diffusivities. 


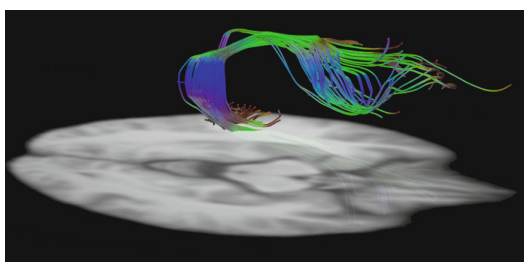

(a)

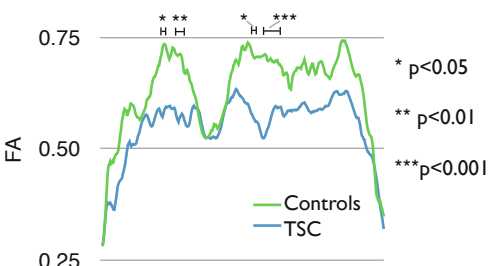

(b)

Fig. 3. (a) Arcuate fasciculus, a set of fibers involved in language, on which tract based statistics was performed, (b) The FA profile in TSC patients shows significantly disrupted white matter fascicules in different clusters, indicated by the stars.

\section{Implementation and Complexity}

The developed methods were integrated in the efficient block matching registration algorithm described in [9]. The parameters used are the following: 4 pyramid level, 10 iterations per level, block size: $5 \times 5 \times 5$, outlier removal rate: $20 \%$. The implementation was multi-threaded. On a 8 core workstation, with $220 \times 220 \times 176$ two-fiber images, the entire registration takes 1.5 hour. All weighted combinations were computed until complete convergence of the soft clustering. The average number of iterations required for that convergence is 4 .

\section{Results}

The registration was applied to a clinical dataset of 45 subjects, 13 controls and 32 patients with tuberous sclerosis complex (TSC), a rare genetic disease associated with impaired white matter integrity. A DTI and a multi-tensor model with three components (one isotropic and two anisotropic) were reconstructed for each subject $[13$.

\subsection{Validation}

An alternative to the method presented in this paper would be to select one of the two tensors in each voxel (e.g. the one with the highest FA) and to perform single tensor registration on this image. Here, we validate that our method works better than this simple alternative. The quality of the alignment is assessed by the sum of square differences of each eigenvalue after alignment of control subjects. Indeed, while the diffusivities can significantly differ in diseased brain, they are approximately equal for healthy subjects. We performed 26 randomly chosen registrations with four levels of regularization, totalizing 104 registrations. In each voxel, the eigenvalues were averaged between the two anisotropic components (weighted by their fractions). Results show that multi-fiber registration performs significantly better than single tensor registration (Fig. 1). 


\subsection{Atlas Construction}

An atlas was constructed using our registration and alternating three steps: aligning all subjects to the current atlas (initially a randomly chosen subject), averaging the aligned subjects (using the weighted combination of mixtures), applying the mean inverse field to the resulting average [14. This atlas remarkably shows areas where multiple fibers are consistently present in all subjects (Fig. 2).

\subsection{Morphometry}

The clinical hypothesis according to which there is substantial white matter shrinkage in TSC subjects was tested by performing a one-tailed two sample ttest on the log-Jacobian of the deformation fields [15. The subject classes were then randomly permuted 4000 times to assess the null distribution of extreme t-scores. The entire process was repeated with single tensor images. As a result, multi-fiber registration reveals more white matter differences $(>3800$ significant voxels) than single tensor ( $<1000$ voxels) (Fig 2 2$)$. The entire process was then repeated with DT-REFinD, a state-of-the art DTI registration algorithm [11, to test whether the improved detection of differences is truly due to the knowledge brought by multi-fiber models. Again, DT-REFinD did not capture all the differences detected by multi-fiber registration ( $<1300$ voxels) (Fig 2) .

\subsection{Tract-Based Statistics}

Some structural subnetworks are believed to be impaired in TSC patients. To test this hypothesis, we analyzed the FA profile along the median tract of the arcuate fasciculus, generated on the atlas by a probabilistic tractography algorithm [5] (Fig. 3(a)). A one-tailed two-sample t-test was performed at every location. A threshold $t_{0}$ was then set to the $t$-statistics and the length of the contiguous supra-threshold segments were recorded. The null distribution of these lengths was assessed by randomly permuting the subjects classes 4000 times. The operation was repeated for a wide range of thresholds $\left(1.5 \leq t_{0} \leq 4.5\right)$ to estimate the robustness of our findings. For $t_{0}=2.7\left(p_{0}=0.01\right)$, with our multifiber registration, four significant clusters, together representing $15 \%$ of the tract were detected, indicating a strong impairment of this subnetwork in TSC patients (Fig. 3(b)). These findings were robust to the choice of $t_{0}$ for any $t_{0} \leq 3.4$. In contrast, single tensor registration only revealed one cluster representing $5 \%$ of the fiber, which was not robust outside the range $1.9 \leq t_{0} \leq 2.8$.

\section{Conclusions}

This paper introduced a registration and atlas construction method to align multi-fiber models. A proper interpolation method and a robust similarity metric were presented. Results in both morphometry and tract-based statistics demonstrated that multi-fiber registration reveals more group differences than DTI 
registration. We therefore believe that this registration method opens new doors to understanding brain disorders based on multi-fiber models.

Acknowledgments. MT thanks the FNRS, BAEF and WBI for their financial support. This work was supported in part by NIH grants R01 EB008015,R01 LM010033, R01 EB013248, and P30 HD018655 and by the Boston Children's Hospital Translational Research Program.

\section{References}

1. Zhang, H., Avants, B., Yushkevich, P., Woo, J., Wang, S., McCluskey, L., Elman, L., Melhem, E., Gee, J.: High-dimensional spatial normalization of diffusion tensor images improves the detection of white matter differences: an example study using amyotrophic lateral sclerosis. IEEE TMI 26(11), 1585-1597 (2007)

2. Minati, L., Weglarz, W.: Physical foundations, models, and methods of diffusion magnetic resonance imaging of the brain: A review. Concepts in Magnetic Resonance Part A 30(5), 278-307 (2007)

3. Barmpoutis, A., Vemuri, B.C., Forder, J.R.: Registration of High Angular Resolution Diffusion MRI Images Using 4th Order Tensors. In: Ayache, N., Ourselin, S., Maeder, A. (eds.) MICCAI 2007, Part I. LNCS, vol. 4791, pp. 908-915. Springer, Heidelberg (2007)

4. Yap, P., Chen, Y., An, H., Yang, Y., Gilmore, J., Lin, W., Shen, D.: Sphere: Spherical harmonic elastic registration of hardi data. NeuroImage 55(2), 545-556 (2011)

5. Bergmann, O., Kindlmann, G., Peled, S., Westin, C.: Two-tensor fiber tractography. In: IEEE International Symposium on Biomedical Imaging, pp. 796-799 (2007)

6. Assaf, Y., Basser, P.: Composite hindered and restricted model of diffusion (charmed) mr imaging of the human brain. Neuroimage 27(1), 48-58 (2005)

7. Taquet, M., Scherrer, B., Benjamin, C., Prabhu, S., Macq, B., Warfield, S.: Interpolating multi-fiber models by gaussian mixture simplification. In: IEEE International Symposium on Biomedical Imaging (2012)

8. Banerjee, A., Merugu, S., Dhillon, I., Ghosh, J.: Clustering with bregman divergences. The Journal of Machine Learning Research 6, 1705-1749 (2005)

9. Commowick, O., Arsigny, V., Isambert, A., Costa, J., Dhermain, F., Bidault, F., Bondiau, P., Ayache, N., Malandain, G.: An efficient locally affine framework for the smooth registration of anatomical structures. MedIA 12(4), 427-441 (2008)

10. Ruiz-Alzola, J., Westin, C., Warfield, S., Alberola, C., Maier, S., Kikinis, R.: Nonrigid registration of 3d tensor medical data. MedIA 6(2), 143-161 (2002)

11. Yeo, B., Vercauteren, T., Fillard, P., Peyrat, J., Pennec, X., Golland, P., Ayache, N., Clatz, O.: Dt-refind: Diffusion tensor registration with exact finite-strain differential. IEEE Trans. on Medical Imaging 28(12), 1914-1928 (2009)

12. Taquet, M., Macq, B., Warfield, S.: A generalized correlation coefficient: Application to dti and multi-fiber dti. In: IEEE MMBIA, pp. 9-14 (2012)

13. Scherrer, B., Warfield, S.: Toward an accurate multi-fiber assessment strategy for clinical practice. In: IEEE International Symposium on Biomedical Imaging, pp. 2140-2143 (2011)

14. Guimond, A., Meunier, J., Thirion, J.: Average brain models: A convergence study. Computer Vision and Image Understanding 77(2), 192-210 (2000)

15. Ashburner, J., Hutton, C., Frackowiak, R., Johnsrude, I., Price, C., Friston, K.: Identifying global anatomical differences: deformation-based morphometry. Human Brain Mapping 6(5-6), 348-357 (1998) 